\title{
Dualité des conventions de la valeur et marchandisation de l'université.
}

\section{Duality of value conventions and commodification of university}

\author{
Hugo Harari-Kermadec et Raphael Porcherot \\ IDHES - Ecole Normale Supérieure Paris Saclay \\ 61 avenue du Président Wilson 94235 Cachan \\ hugo.harari@ens-paris-saclay.fr \\ raphael.porcherot@ens-paris-saclay.fr
}

Résumé : Si la marchandise capitaliste est la forme dominante de la richesse dans la société contemporaine, elle n'est pas exclusive. À partir de l'hypothèse que l'université, après d'autres services publics, subit des transformations qui l'amène à s'inscrire dans cette forme dominante, nous suivons les dispositifs qui soutiennent cette évolution. Nous nous inscrivons dans une approche constructiviste de la valeur, qui doit être produite et soutenue comme représentation de la richesse pour permettre l'émergence, au niveau institutionnel, d'un marché de l'enseignement supérieur et la recherche, mais aussi se déployer au sein même des établissements pour réorganiser la production. Ce sont les dispositifs de quantification du travail universitaire que nous proposons d'analyser ici comme vecteurs d'extension du fétichisme de la marchandise sur la production et la transmission des connaissances.

Abstract : The capitalist commodity is the dominant form of wealth in the current society, but not the only one. We use the commodification process of higher education and research as an experiment to observe the devises that support such a transformation of social form. This is then a constructivist approach of value: the value has to be constructed and supported has representation of wealth to product material effects. If value is central for the market, at an institutional level, it's on the productive sphere that we mainly track its construction and effects, within organisations. We study the devises of quantification of academic work as means of extension of the commodity fetishism on the production and transmission of knowledge. 
Mots-clés: Fétichisme de la marchandise, quantification, université, marchandisation, valeur Keywords: Commodity fetishism, quantification, university, commodification, value

\section{Introduction}

Au début du XXle siècle, en France comme aux échelles européenne et globale, l'université est prise dans une série de transformations profondes. Les pays anglo-saxons semblent avoir pris une avance importante dans ce processus qui conduit à l'« acte de décès du service public d'enseignement supérieur en Angleterre » [Vinokur et Eyraud, 2018]. La hausse des frais d'inscription, accompagnée d'une explosion de la dette étudiante, est l'aspect le plus visible et le plus inquiétant de cette dynamique. Plusieurs approches critiques sont possibles. On peut légitimement craindre un renforcement des inégalités d'accès à l'enseignement supérieur [Flacher et Harari-Kermadec, 2013 ; Le Breton et Harari-Kermadec, 2013]. Dans le capitalisme financiarisé contemporain, on peut également défendre que c'est la vente à crédit qui est le trait saillant de la marchandisation de l'enseignement supérieur, parce qu'elle conduit d'un côté à un asservissement à long terme de la future main d'œuvre [Lazzarato, 2011] et à une création massive de capital fictif [Durand, 2014]. Cet article propose d'adopter un regard plus large, qui se ne cantonne pas à observer l'effet de la marchandisation sur les seul·es étudiant·es. Il s'agit plutôt de se saisir de l'université comme «terrain de choix pour étudier les dynamiques [...] du capitalisme financiarisé » [Lebaron, 2015] et y suivre la constitution d'une nouvelle marchandise, cette « chose extrêmement embrouillée, pleine de subtilités métaphysiques et de lubies théologiques » [Marx, 1867]. Pour Orléan [2005] ce sont précisément les propriétés miraculeuses de cette «chose » qui justifie l'unité entre la sociologie et l'économie : s'il s'agit de comprendre comment les relations marchandes peuvent se fonder sur la médiation des objets plutôt que sur les rapports interpersonnels.

Cette enquête qui ne peut se limiter au marché lui-même, à l'échange - en langage marxien, à la sphère de la circulation ; mais doit s'étendre à la sphère de la production, et donc au travail dans l'université. C'est qu'en se restreignant au marché, on risque de ne penser que la modification des modalités d'accès aux formations et de perdre de vue l'essentiel [Szadkowki, 2016]. La marchandisation de l'université passe par une transformation du travail universitaire pour l'objectiver et pour l'orienter vers une nouvelle finalité, la valorisation du capital, l'université étant ainsi insérée sans médiation dans le circuit du capital. Ainsi, le processus de marchandisation doit être étudié à la jonction des deux sphères de la production et de la circulation, le but étant de déterminer le rôle de la monétisation de l'accès aux formations dans la transformation de l'université en unité de production de services marchands. Cette transformation se déroule sur deux plans : au niveau organisationnel, le processus concret de production - le 2 sur 20 
travail académique - doit évoluer pour répondre progressivement à une demande solvable plutôt que pour fournir un service public, et se réorganiser pour dégager des profits ; au niveau institutionnel, le déploiement des représentations qui renouvellent le sens et l'existence sociale du travail académique [Hall et Bowles, 2016] peut alors se lire comme un investissement de forme [Thévenot, 1989]. L'approche retenue dans cette article s'inscrit donc dans ce que Sobel [2016, p. 62] appelle une interprétation constructiviste du fétichisme de la marchandise : «le mode d'existence des rapports sociaux du capitalisme ». Comment l'enseignement supérieur change-til de mode d'existence ? Notre ambition est de nous appuyer sur les outils de la sociologie de la quantification [Desrosières, 1993, 2008a, 2008b] pour suivre comment de nouvelles représentations émergent et participent à redéfinir l'université. À partir de différents travaux portant sur l'enseignement supérieur en France et au Royaume-Uni principalement, nous cherchons à développer un cadre théorique permettant d'appréhender les différentes évolutions contemporaines de l'université comme participant d'un même processus de marchandisation. Les frais d'inscription s'insèrent alors dans un vaste ensemble de dispositifs d'évaluation quantitative, parfois monétaire et parfois non, qui deviennent dominants sur le plan idéel, dans la façon dont on conçoit l'université, y travaille et y étudie. Dans l'approche constructiviste, le plan idéel des représentations n'est pas une apparence apposée sur une réalité matérielle préexistante et plus profonde. Représentations et matérialité interagissent constamment : d'une part, les représentations marchandes ont besoin pour s'imposer de s'appuyer sur un pouvoir bien matériel ; d'autre part, le processus de production, le travail, ne peut s'organiser qu'à partir de conceptions sociales, de conventions qui permettent la coordination des producteurs entre eux, et avec les consommateurs au niveau du marché. Enfin, la progressive marchandisation de l'université est également une inscription du travail à l'université en travail abstrait, c'est-à-dire en activité dont la valeur sociale est économique et validée par le marché. Plus encore, dans l'économie marchande capitaliste, le travail abstrait ayant pour raison d'être de produire de la survaleur, la valeur du travail doit excéder la valeur de la force de travail. Ici encore, le fait de saisir la transformation en cours de route permet d'observer la concurrence entre les conventions de valeur antérieures, que l'on peut décrire en suivant Harribey [2013] comme une valeur monétaire non marchande, et les conventions de valeur émergentes qui conduisent à la valeur d'échange et au prix.

Si le système français d'enseignement supérieur français est loin d'être totalement transformé en secteur marchand [Lamarche, 2003], il est l'objet d'un intense processus de transformation depuis le début du siècle. Après une première phase de transformation principalement inscrite à l'échelle européenne [Bruno, 2008], c'est l'émergence d'un marché global de l'enseignement supérieur symbolisé par le classement de Shanghai qui légitime les injonctions réformatrices [Harari-Kermadec et Moulin, 2015]. Un marché global bien réel, dominé par les Etats-Unis et le Royaume-Uni, talonnés par l'Australie qui capte une large part de la 3 sur 20 
demande croissante issue de l'explosion du nombre d'étudiant.es asiatiques en migration internationale [Min et Falvey, 2018]. Cette extension concomitante de la marchandisation et d'une nouvelle phase de globalisation de l'université invite à mettre en regard les dispositifs français avec leurs équivalents étrangers [de Verdalle et al., 2012]. S'il ne faut pas voir dans les cas anglosaxons comme un destin inéluctable pour le système universitaire français, le mouvement de marchandisation est bien global en ce qu'il affecte tous les pays, avec des spécificités nationales décisives dans la transposition organisationnelle des logiques de valorisation et dans les formes de résistance qui leur sont opposées. Nous soutiendrons en effet que l'importation de ces dispositifs s'accompagne de celle de l'idéologie qui les sous-tend. Le plan «Bienvenue en France » présenté le 19 novembre 2019 par le Premier Ministre Edouard Philippe, qui prévoit une hausse spectaculaire des frais d'inscription dans les diplômes nationaux pour les seul·es étudiant·es extra-européen·nes, incarne parfaitement cette articulation de la globalisation et de la marchandisation dans les politique publique de l'enseignement supérieur.

L'article s'organise en deux parties : dans un premier temps, nous étudierons les dispositifs de normalisation et de classement qui participent à mettre en place et structurer un marché de l'enseignement supérieur en en proposant une représentation quantitative et concurrentielle. Dans un second temps, ce sont les dispositifs d'évaluation du travail universitaire, produisant de l'enseignement aussi bien que de la recherche, qui nous intéresseront. Nous défendrons l'hypothèse que le rôle croissant de ces modalités quantitatives de management du travail universitaire entraîne une compétition entre deux modes de validation sociale de la valeur de ce travail, le mode émergent étant proprement capitaliste.

\section{Instituer un marché de l'enseignement supérieur}

Pour être vendu et acheté comme toute marchandise, l'enseignement supérieur doit s'inscrire dans un espace suffisamment stable et homogène pour permettre l'établissement de prix. La sociologie de la quantification et l'économie des conventions se sont largement intéressées aux dispositifs soutenant ces prix : Desrosières et al. [2013] réunissent de nombreuses contributions portant sur des biens divers, allant de la viande à l'électroménager. Si les prix des marchandises agricoles ou manufacturées se révèlent beaucoup plus administrés que la théorie économique standard le prétend, l'émergence de prix pour les services d'enseignement supérieur cumule les difficultés identifiées pour les services d'une part et les biens culturels d'autre part. Dans les services, la normalisation nécessaire à la vente ne peut se faire qu'« au prix d'hypothèses héroïques dans des productions pourtant marquées par leur singularité, par une qualité variable et des processus de production peu standardisables » [Jany-Catrice, 2012]. Pour les biens culturels, la difficulté réside dans le fait qu'on ne « consomme » le bien qu'une fois et que c'est au cours de la consommation que la qualité réelle est révélée. La marchandisation de l'enseignement 4 sur 20 
supérieur consiste donc en la construction d'un marché des singularités [Karpik, 2007] qu'il s'agit de baliser à l'aide d'une forme objectivée de la qualité académique, suivant des dimensions fixées et des nomenclatures standardisées [Boulet, 2013, p. 41].

Les classements d'établissements sont les principaux dispositifs donnant une telle représentation objectivée (mais relative) de la qualité. Ils structurent l'espace (ou les espaces lorsqu'ils sont disciplinaires par exemple) de l'enseignement supérieur. Cette partie s'intéresse à la production de catégories d'établissements par le classement de Shanghai avant d'analyser grâce aux travaux d'Espeland et Sauder [2007] comment les classements instituent autant (plus ?) qu'ils ne décrivent un espace concurrentiel d'offre d'enseignement supérieur.

\section{A. Produire des conventions pour construire un marché}

Depuis le milieu des années 2000, la légitimation des transformations du système universitaire français est souvent externe et globale : le rang de la France serait menacé, tant en terme de qualité de la recherche que d'attractivité des étudiant.es en migration internationale. Dans le contexte de l'autonomie, les établissements français sont alors interpelés à deux échelles simultanément : par leurs tutelles nationales mais au nom d'une concurrence globale. Le classement de Shanghai s'est avéré être parfaitement adapté pour une telle interpellation puisqu'il permet de faire surgir cette menace globale à la fois dans le débat national et à l'échelle des établissements [Barats et Leblanc, 2013 ; Barats et al., 2018]. Pour produire un classement d'universités, il faut disposer ou convenir d'une population. Le classement de Shanghai ne porte pas sur une telle population d'universités, définie de façon homogène et préalable. Le classement institue donc une population d'universités à l'échelle internationale, mais uniquement partielle puisqu'il ne classe qu'une faible partie des établissements (on trouve par exemple 33 établissements français sur le site du classement de Shanghai, sur les 142 listés sous le Titre ler du livre 7 du code de l'éducation). En France tout du moins, il participe à donner corps à une catégorie d'établissements, les universités de recherche de classe mondiale ("Vers de nouvelles universités de recherche de classe mondiale », Mercredi 13 Mai 2015, conférence organisée par Thierry Coulhon, président de l'Université Paris Sciences et Lettres, ex-directeur du programme « campus d'excellence » du gouvernement Fillon et futur conseiller du Président Macron pour les questions universitaires). Les regroupements d'établissements promus par une série d'initiatives réglementaires et d'incitations budgétaires (pôles régionaux, Idex, Comue, EUR, etc.) dans les années 2000 et 2010 restructurent les établissements suivant une échelle mieux adaptée au classement [Supiot, 2015, p. 249] : il s'agit d'obtenir une visibilité internationale. S'il est caricatural de penser le classement de Shanghai comme étant à l'origine de ces regroupements, il aide à penser un paysage universitaire français inséré dans une compétition internationale et à distinguer les établissements qui y participent de ceux qui restent en dehors. C'est autour de cet enjeu de 5 sur 20 
redéfinition et de catégorisation de l'enseignement supérieur français qu'une controverse éclate au sein de la Conférences des Présidents d'Université (CPU), allant jusqu'au dépôt de deux listes concurrentes pour présider la CPU. Les présidents de 21 universités hors course au classement de Shanghai signent ainsi le 29 mai 2015 une tribune collective intitulée « Quel avenir pour l'Enseignement Supérieur et la Recherche français ? » dénonçant cette évolution : « L'obsession des classements internationaux prévaut dans les choix de soutien budgétaire, la notion d'excellence scientifique et de masse critique, deviennent centrales au détriment des autres missions de l'université dont la réussite pour tous ou la formation tout au long de la vie. » La production de la catégorie des universités de recherche de classe mondiale caractérise les établissements autant en positif (« excellence scientifique » et « masse critique ») qu'en négatif (« la réussite pour tous » et la «formation tout au long de la vie » sont délaissées). On assiste ici à un processus de redéfinition de ce que devrait être une université en France, ou plutôt à une bicatégorisation progressive, l'attribution des distinctions et des budgets de type ldex participant à renforcer symboliquement et financièrement la démarcation entre la catégorie des universités d'excellence et le reste des établissements. Mais, on le lit dans la tribune des 21 présidents, cette évolution ne va pas sans résistance, et est loin d'être aboutie et stabilisée.

Tant que la légitimité de ces catégories et des classements internationaux reste contestée, la première phase de la quantification (pour Desrosières [2008b], quantifier c'est convenir et mesurer) reste ouverte, jusqu'à la stabilisation d'une configuration combinant la répartition du pouvoir politique et des moyens, les représentations quantitatives, la nomenclature juridique et la réalité des établissements. Dans l'attente, les classements ne permettent pas réellement de baliser un marché de l'enseignement supérieur, les étudiant·es faisant encore largement appel à d'autres prescripteurs, familiaux [Chauvel et Clément, 2014] ou amicaux [Frouillou, 2014]. Les conventions traditionnelles restent donc dominantes, du moins dans les représentations des étudiant·es présent·es dans les établissements français au début des années 2010. Elles renvoient davantage à une lecture de la qualité en termes de réputation qu'en termes d'excellence [Paradeise et Thoenig, 2015]. Les conventions de valeur proprement économique sont encore peu développées, d'autant que pour l'essentiel l'accès au supérieur en France [Cour des comptes, 2018] n'est pas payant (80\% des étudiant·es sont boursier.es ou paient les frais correspondant aux diplômes nationaux, de l'ordre de quelques centaines d'euros) : ou du moins par encore, les formations payantes étant en forte croissance. On peut également s'interroger sur l'effet de la mise en oeuvre de la plateforme de candidature Parcoursup sur la destabilisation des conventions traditionnelles, dès lors que cette plateforme organisant l'essentiel de l'accès aus supérieur met en avant des indicateurs quantitatifs tels que les taux d'accès aux différentes formations. 


\section{B. L'orientation scolaire réduite à une demande de service ?}

Si le classement de Shanghai pousse en direction d'une définition globale et concurrentielle des universités de recherche qui participe à préparer la marchandisation en France [Eloire, 2010], il est bien moins mobilisé dans d'autres contextes. Aux États-Unis, nul besoin de faire appel à la globalisation pour justifier la mise en concurrence de l'enseignement supérieur. C'est donc directement au niveau national que les classements font sens et ils disposent déjà d'une catégorisation, la liste d'établissements accrédités par les instances de régulation. Le journal U.S. News and World Report (USN) peut ainsi entreprendre une série de mesures communes sur l'ensemble des écoles de droits (Law schools) accréditées afin d'établir son classement thématique. Après différentes évolutions, I'USN classe l'ensemble des 190 établissements habilités en trois catégories : les 100 premières Law schools sont classées de la première à la 100e, les restantes (101 - 190) étant réparties entre deux catégories (intermédiaire et inférieure). Selon Espeland et Sauder [2007], ce classement énonce une forme de prophétie auto-réalisatrice, ou plutôt auto-renforçante puisque le classement contribue activement à stabiliser la hiérarchie qu'il est censé décrire : "Rankings reproduce and intensify the stratification they are designed to measure. » [Espeland et Sauder, 2007, p. 14].

Le premier mécanisme d'auto-réalisation est l'impact du classement d'une année sur celui de l'année suivante. Face à la difficulté de classer près de 200 établissements (et leur absence d'information sur la plupart d'entre eux), les universitaires et professionnels du droit interrogés par I'USN pour évaluer la réputation des Law schools se basent en grande partie sur le classement USN de l'année précédente. Mais surtout, le classement se renforce par son rôle effectif dans l'orientation de la demande de services d'enseignement supérieur en droit aux Etats-Unis. En orientant les étudiant·es, il oriente les frais d'inscription et donc les moyens. Aux frais d'inscription s'ajoutent les dons des alumni, qui réagissent en cas de chute dans le classement, "your monetary support from alumni say 'Well, I'm not sure I want to support a school that's going in the wrong direction", and your money starts to dry up " [Sauder et Lancaster, 2006, p. 130]. La chute dans le classement entraîne alors une réduction de moyens qui réduit d'autant les possibilités de réaliser un enseignement de qualité. Laval et al. [2012, p. 156] développe la même idée pour le classement de Shanghai : "Le mécanisme des palmarès de type Shanghai pousse à cette spirale inflationniste: plus l'entreprise universitaire est richement dotée, plus elle est en mesure de s'acheter des "stars" de la recherche qui feront augmenter la cote de l'université, et plus il sera facile d'élever les frais d'inscription. " La hiérarchie énoncée par le classement ne reste pas idéelle, elle s'inscrit dans la réalité par sa capacité à se refléter dans les financements à la disposition des établissements.

On peut analyser ce mécanisme d'auto-renforcement comme l'adaptation d'une convention de valeur sur l'autre. La valeur académique, non économique, identifiée à la réputation, s'exprime 7 sur 20 
dans le classement sous une forme quantitative, ordinale. Le mécanisme marchand d'accès à l'enseignement supérieur aux Etats-Unis contraint les prix (les frais d'inscription) à refléter, au moins partiellement, le classement. Le mécanisme de financement des établissements, par ces frais et par les dons, boucle le circuit: les financements obtenus jouent en retour sur la valeur académique. Ce phénomène d'auto-renforcement rapproche donc les deux jeux de conventions de valeurs (académiques et économiques) l'un de l'autre. Les conventions de valeur académique utilisées dans le classement, même si elles font appel à la réputation, ne sont pas pour autant traditionnelles au sens de la section précédente : il s'agit bien d'évaluer les établissements en vue de les classer tous à l'aide d'une unique formule portant en elle une définition de l'excellence.

Le classement USN repose sur quatre critères (réputation, sélectivité, placement et moyens de l'établissement), chacun étant mesuré de façon identique pour chaque établissement et combinés à l'aide d'un jeu de pondération (respectivement $40 \%, 25 \%, 20 \%$ et $15 \%$ ). Une fois les quatre critères combinés, tous les établissements sont donc représentés sur un axe unique, qui exprime la qualité des Law schools de façon quantitative et unidimensionnelle. Cette étape d'agrégation produit une nouvelle relation entre les Law schools, à la fois précise et globale, qui se traduit en positions relatives dans le classement. Le journal propose ainsi aux étudiant.es de choisir l'établissement le mieux classé parmi ceux qui sont accessibles : ceux dont les exigences en termes de capitaux scolaire (résultats aux examens LSAT de fin du secondaire étatsunien) et économique (capacité d'endettement) ne constituent pas des barrières à l'entrée. Ce choix est autrement plus simple qu'un choix basé sur une réputation multidimensionnelle et évasive [Paradeise et Thoenig, 2015], et plus adéquat à mettre en œuvre qu'un calcul financier à l'heure de contracter un prêt étudiant. Le dispositif que constitue le classement USN apparait alors comme participant à reconfigurer les conventions de valeur académique pour les adapter à une cadre marchand. Mais l'identification n'est pas parfaite: les dons des alumni ne correspondent pas à un achat, ils renvoient bien plus à des relations interpersonnelles qu'à des relations par la médiation des objets pour reprendre la différenciation d'Orléan [2005] présentée dans l'introduction.

Certains établissements français apparaissent également dans les classements médiatiques thématiques que l'on peut rapprocher du classement USN : le classement du Financial Times pour les Busines Schools, celui du Times Higher Education pour les MBA ou les classements nationaux de l'Etudiant. Les grandes écoles de commerce, et depuis quelques années les écoles d'ingénieur qui pratiquent des frais élevés, peuvent alors être impactées par ces classements médiatiques, et modifier leur pratique pour s'y adapter [Blanchard, 2011 ; Delespierre, 2016]. Mais contrairement à ces classements, le classement de Shanghai n'est pas appuyé en France par une matérialisation par le marché puisque le poids des frais d'inscription reste encore extrêmement réduit dans les budgets des universités françaises. La dualité grandes écoles/universités semble limiter l'extension de la marchandisation.

8 sur 20 


\section{Les universités reprennent les conventions du marché pour proposer une offre adaptée}

Avant de s'inscrire dans la conscience sociale comme allant de soi, comme mode d'existence naturel des phénomènes quantifiés, un classement n'a d'effet que « parce qu'il est soutenu par l'institution qui lui donne une force ou une efficacité immédiate ». C'est, selon Ambroise [2015, p. 32], le sens de la performativité d'un énoncé. Si le classement de Shanghai est performatif et pousse les établissements français à réagir lorsque la position qui leur est attribuée ne correspond pas à l'excellence internationale revendiquée, c'est donc parce qu'il sert de référence aux responsables politiques, aux jurys attribuant des financements de type Idex et aux chefs d'établissements. Sur le long terme cependant, le constat de cette efficacité et sa répétition peuvent conduire à ce que le classement acquière une légitimité de fait, une certaine autonomie, que la controverse autour de son mode de calcul s'estompe et finalement qu'il puisse se passer du soutien permanent des instances de direction de l'ESR. La phase active de convention est alors, pour un temps au moins, achevée, et la quantification se réduit à une mesure, à l'application consensuelle des modalités techniques de classement.

La capacité des classements d'établissements à accéder au statut de carte fidèle de l'espace universitaire est renforcée par deux mécanismes au moins. Premièrement, l'effet d'autoréalisation des classements décrit par Espeland et Sauder [2007] et rappelé ci-dessus réorganise l'espace universitaire conformément au classement : si la répartition des moyens financiers et du capital scolaire suit le classement, alors celui-ci finit par décrire la hiérarchie réelle. La prophétie s'avère juste parce que la réalité se transforme pour lui correspondre. Deuxièmement, le classement parvient à redéfinir plus intimement les établissements classés en leur faisant adopter ses critères, en s'érigeant lui-même en convention académique dominante. Ainsi, parce que le classement USN mesure la sélectivité des établissements (second critère) sur la base des résultats des lycéen·nes aux LSAT, une donnée quantitative homogène et disponible, les établissements revoient leurs propres critères de recrutement dans ce sens. D'autres critères, plus qualitatifs et distincts d'un établissement à l'autre, par exemple l'adéquation entre la vocation du candidat et celle de l'établissement évaluée lors d'un entretien, perdent en importance. Les politiques de bourses sont également revues dans le sens du classement : en remplaçant les bourses sur critères sociaux par des bourses sur critères académiques, les établissements peuvent améliorer le score LSAT moyen des lycéen·nes admis·es et donc améliorer le rang de l'établissement dans le classement USN. La définition implicite d'une bonne Law school portée par le classement (un établissement dont la population étudiante dispose d'une moyenne LSAT élevée) se concrétise à mesure que son autorité s'impose. Les conventions académiques, évoluant pour se rapprocher des conventions économiques, produisent donc des 
effets au-delà du seul marché (les représentations des potentiel-les étudiant·es) : les pratiques dans les établissements (ici de sélection) s'adaptent également.

Cette première analyse des classements d'établissements a cherché à montrer comment la quantification propose des repères cognitifs qui peuvent progressivement s'imposer comme des descriptions adéquates de la réalité, quitte à ce que ce soit la réalité qui évolue. L'existence d'un marché et de prix peut être déterminante dans le mécanisme d'auto-réalisation du classement comme c'est le cas pour le classement USN des Law Schools. L'effectivité du classement peut également s'appuyer sur une autorité externe, celle des responsables politiques et des chefs d'établissement comme on peut l'observer pour le classement de Shanghai en France.

On l'a dit, la référence au classement de Shanghai peut être stratégique [Gingras, 2013, p. 105] et servir un agenda de réformes antérieur à la création du classement. Les réformateurs donnent en retour un pouvoir accru aux classements, par la publicité qu'ils leur offrent (même lorsqu'ils formulent des réserves, [Barats et Leblanc, 2013]), mais aussi parce que les réformes vont dans le sens d'une mise en concurrence et en marché de l'enseignement supérieur, ce qui est favorable, on l'a vu, à étendre l'impact des classements sur les étudiant.es. C'est dans ce contexte que les chefs d'établissement s'intéressent aux classements, qui les impactent à de multiples niveaux : par transmission de l'importance qu'y accorde la tutelle mais aussi, une fois les réformes engagées, par la nécessité de placer leur établissement sur ce marché. Selon I'historien des sciences Yves Gingras: «On peut comprendre le comportement des dirigeants face aux classements si l'on note qu'ils servent essentiellement au marketing des universités. L'OCDE fait la promotion, depuis au moins les années 1990, d'un marché mondial de l'éducation supérieure, et l'émergence des classements mondiaux au début des années 2000 s'inscrit dans ce courant néolibéral qui fait des universités un marché comme un autre. » [Gingras, 2013, p. 98]. On peut en effet faire l'hypothèse que l'adaptation des établissements français les plus dotés en capitaux de toutes sortes à ce classement (où ils cohabitent avec des établissements aux frais d'inscription très élevés), accompagnée par des politiques publiques de mise en concurrence, participe à produire les conditions d'émergence d'un marché des services d'enseignement supérieur.

\section{Changement organisationnel par les dispositifs d'évaluation et de valorisation du travail académique}

Les travaux d'économie des conventions et de sociologie de la quantification auxquels nous avons fait référence jusqu'ici se sont principalement intéressés au rôle des indicateurs quantitatifs sur le marché. L'analyse de l'effet de la quantification sur les pratiques des universitaires est davantage traitée dans une littérature anglo-saxonne à partir de catégories issues du marxisme [Hall et Bowles, 2016 ; Szadkowski, 2016; Harvie, 2006]. C'est que l'achat et la vente de service 10 sur 20 
marchand d'enseignement supérieur ne peut se faire en laissant intacte l'activité académique ellemême. Pour Marx, une configuration très spécifique du travail dans la sphère de la production est la condition de l'échange marchand sous le capitalisme. L'existence de prix ne fait que manifester qu'un bien ou un service est une marchandise, c'est-à-dire qu'il est le produit d'une part du travail sous son mode d'existence capitaliste: «Ce n'est pas la monnaie qui rend les marchandises commensurables : au contraire. C'est parce que les marchandises en tant que valeurs sont du travail matérialisé, et par suite commensurables entre elles, qu'elles peuvent mesurer toutes ensemble leurs valeurs dans une marchandise spéciale, et transformer cette dernière en monnaie, c'est-à-dire en faire leur mesure commune. » [Marx, 1867].

Dans cette partie, nous cherchons à suivre l'émergence de cette condition nécessaire au développement d'un marché de l'enseignement supérieur : comment l'enseignement supérieur devient-il une marchandise, comment le travail académique devient-il producteur de marchandises, c'est-à-dire du travail tel qu'il existe dans l'économie capitaliste ?

\section{A. Une valeur monétaire non marchande du travail universitaire}

Pour s'engager dans la marchandisation, il faut que le travail académique quitte son mode d'existence social antérieur. Dans sa configuration traditionnelle, s'il n'est pas capitaliste, le travail universitaire n'est pas pour autant sans valeur économique. Selon Harribey [2013, p. 17], il est producteur de valeur, mais d'une valeur monétaire non marchande, exactement égale à la valeur de la force de travail qui l'a produit, c'est-à-dire au salaire. Pour devenir marchande, cette valeur du travail doit se distinguer de la valeur de la force de travail. D'un point de vue conventionnel, il faut déstabiliser le jeu de conventions de l'emploi pour permettre le déploiement de deux séries de conventions (partiellement) autonomes, les unes se référant aux universitaires (valeur de la force de travail) et les autres à leurs produits d'enseignement et de recherche (valeur produite par le travail). Le travail académique doit ensuite être incorporé au mode d'existence dominant du travail sous le capitalisme, ce que Marx désigne comme du travail abstrait. Les services produits par le travail académique deviennent alors commensurables avec les autres marchandises capitalistes, et peuvent donc exprimer leur valeur en monnaie.

Selon Harribey [2013, p. 17], les universitaires, comme les autres salarié.es de la fonction publique, produisent de la valeur monétaire, mais non marchande. En effet, «les employés de l'État ne sont pas des salariés identiques à ceux employés par les capitalistes. Ils échangent leur force de travail contre un salaire qui [...] se trouve correspondre, par convention sociale, à l'évaluation monétaire des services nets rendus à la collectivité » [Harribey 2013, p. 373]. La valeur de ce travail, ce qu'il apporte à la société d'un point de vue économique, est donc directement mesurée par ce coût de la force de travail. Du point de vue de la valeur, le travail 11 sur 20 
dans une université non marchandisée n'est donc pas séparé de la force de travail. Cette égalité interdit toute plus-value et donc le lancement d'une accumulation. En développant des mesures de la valeur du travail distinctes de la valeur de la force de travail, le travail peut acquérir une existence sociale autonome, qui ne peut devenir valeur qu'à la condition d'être à nouveau exprimé sous forme monétaire.

Dans une approche constructiviste, cette nouvelle existence sociale doit émerger de dispositifs qui la soutiennent et l'instituent, représentation et matérialité étant deux aspects d'une même réalité qui agissent l'un sur l'autre. Selon Laval et al. [2012, p. 13], c'est le nouveau management public et son usage de l'évaluation qui modifient les activités pédagogiques et scientifiques et produisent une : «forme valeur de la connaissance». Les dispositifs d'évaluation seraient ainsi des outils de transformation de l'enseignement supérieur et de la recherche. Szadkowski [2016, p. 23] fait appel au concept de subsomption formelle pour caractériser comment le nouveau management public inclus le travail universitaire dans le circuit de valorisation du capital. La marchandisation du travail académique peut alors précéder la privatisation des établissements : une transformation de forme permet l'insertion dans un circuit de valorisation alors que le travail reste réalisé en dehors des entreprises capitalistes. Dans la section suivante nous analysons à partir de l'exemple britannique comment la quantification peut produire une telle transformation de forme qui sépare le travail académique de ses spécificités et le rapproche du travail capitaliste dominant.

\section{B. L'évaluation de la recherche au Royaume-Uni : un dispositif d'abstraction du travail académique...}

Le Research Excellence Framework (REF) britannique est un exemple particulièrement abouti de dispositif d'évaluation quantitative de la recherche. II prend en 2009 la suite du Research Assessment Exercise créé dès 1986, et fonde la répartition des subventions publiques pour la recherche à partir d'indicateurs de citations. II agit indirectement sur l'enseignement puisque ses évaluations quantitatives sont reprises dans la presse et la communication des établissements à destination des étudiant.es. Le REF a pour mission l'évaluation quantitative de la productivité, puisqu'il s'agit d'identifier qui « produit le plus de publications et de citations par $£$ de financement public » (site internet et plaquette du REF, 2013, traduits par Boulet [2013]). Concrètement, le REF organise la répartition des subventions publiques au titre de la qualité de la recherche publique sur la base de scores attribués aux départements universitaires (en charge à la fois de l'enseignement et de la recherche). Dans un premier temps, le département est identifié aux productions scientifiques de ses chercheur.ses. Ces productions sont classées suivant 5 qualités de «inférieurs aux standards » à « de pointe mondiale ». À chaque qualité correspond 12 sur 20 
une pondération qui permet de calculer la contribution de chaque article à la qualité globale de la production du département évalué. Le score de qualité de la recherche contribue au score REF final pour deux tiers. Interviennent également une évaluation de l'impact social de la recherche et de son environnement.

L'évaluation de la production de recherche par le REF est une quantification qui, dans sa phase active de convention, produit un effet d'abstraction. En convenant des modalités de la mesure et de ce qui est mesuré, on redéfini le phénomène quantifié sous une forme abstraite, c'est-à-dire générale, débarrassée des spécificités du phénomène. L'usage des nombres en luimême porte une «visée universaliste » pour reprendre l'expression de Desrosières [2008a]. L'évaluation (des établissements, des formations, des laboratoires et des personnel.les) de l'enseignement supérieur et de la recherche, lorsqu'elle s'exprime sous une forme quantitative, requière des opérations préalables « de codification, standardisation et taylorisation des tâches » [Faure, 2005] qui mettent de côté les spécificités et les subjectivités incommensurables. On peut souligner les effets aliénant de cette abstraction [Harvie, 2006], articulée à la perte d'autonomie de la profession universitaire (statut spécifique, gestion de carrière par les pairs) et à la subordination à un management suivant une rationalité comptable et non scientifique. L'abstraction [Hall et Bowles, 2016, p. 38] des conditions concrètes et personnelles du travail académique est aussi une transformation en direction du travail le plus général, sous sa forme dominante dans la société capitaliste, travail abstrait chez Marx, travail producteur de valeur monétaire marchande chez Harribey.

Le REF produit ce type d'effet d'abstraction en plusieurs étapes. Après avoir identifié les unes aux autres des productions d'universitaires différentes, de départements différents, sur des sujets différents, en les catégorisant ensemble, le REF s'affranchit également des frontières disciplinaires. Les départements sont évalués suivant les mêmes critères de publications, d'impact et d'environnement quelle que soit la discipline. Ces trois critères sont combinés suivant les mêmes pondérations $(60 \%, 20 \%$ et $15 \%)$. L'évaluation est menée néanmoins par des experts de chaque discipline, qui doivent trier les publications suivant les 5 catégories de qualité. Mais le poids de la discipline est très réduit : d'une part parce que quelle que soit la discipline, l'évaluation prend la même forme, ce qui suppose que les objectifs soient structurellement identiques ; d'autre part, le volume de publications à évaluer pousse les experts à avoir recours à leur tour à des méthodes quantitatives d'évaluation, à partir d'indices de citations ou de classements de revues ; enfin, parce que les périmètres disciplinaires s'élargissent (et donc le nombre de « disciplines » se réduit). On est ainsi passé entre 2008 et 2014 de 67 à 36 jurys d'évaluation [Boulet et Harari-Kermadec, 2014]. Les experts du REF ne peuvent donc évaluer la qualité du travail scientifique, ce qui demanderait du temps, une connaissance précise du champ de recherche dans un sens étroit et une méthodologie adaptée. C'est une capacité a-disciplinaire à produire des résultats visibles de la recherche qui est finalement mesurée.

13 sur 20 
Du point de vue de la valeur académique traditionnelle, ces modalités d'évaluation sont très insatisfaisantes : en plus de demander une intense activité de visibilisation qui empiète sur le temps disponible pour faire de la recherche ou de l'enseignement, elle est contradictoire avec certaines caractéristiques concrètes du travail universitaire comme les collaborations extérieures au laboratoire, à l'établissement et même au pays concerné par l'évaluation. Enfin, l'évaluation invisibilise le travail des personnels d'appui, bibliothécaires, ingénieur.es, technicien.nes, etc, pourtant nécessaire à la production des publications évaluées. Ces activités apparaissent alors comme de purs coûts, qu'il pourrait être naturel de réduire et d'externaliser. L'expression quantitative et homogène de la production de recherche à l'échelle nationale et à travers les disciplines ouvre la voie à leur expression dans une unité commune et partagée avec les autres secteurs productifs, la monnaie.

\section{C. ...qui débouche sur une expression monétaire permettant sa marchandisation}

La capacité des mathématiques à combiner les nombres invite à sortir de la catégorie dans laquelle les phénomènes ont été mesurés en commun et comparés à une norme pour entrer en relation avec d'autres phénomènes, de plus en plus lointains. L'unité de mesure joue ici un rôle important : elle porte la dernière trace du contexte de la mesure, et c'est donc sa conversion qui permet d'élargir la commensuration. La monnaie sert alors d'unité de mesure universelle qui élargit la commensuration à la société toute entière et propose comme norme commune le retour sur investissement maximal. L'abstraction est alors d'étendue maximale, mais non neutre : l'expression monétaire est économique, la quantification prépare la marchandisation.

L'expression sous forme monétaire du travail est l'unité de mesure qui manifeste l'abstraction de ce travail, le signe du remplacement d'une activité organisée suivant des valeurs traditionnelles multidimensionnelles par un travail produisant sur le plan concret des valeurs d'usage (c'est-àdire n'ayant de sens que dans leur consommation) et sur le plan abstrait de la valeur monétaire marchande. En effet, selon Harribey [2013, p. 124-125], « le travail abstrait ne doit pas être entendu comme une quantité de travail, même débarrassé de ses aspects concrets, mais comme une quantité de monnaie, seule forme dans laquelle il peut s'exprimer en tant que substance de la valeur ». Le passage du Capital sur la monnaie cité plus haut souligne par ailleurs que cette expression monétaire, sous la forme d'un prix lorsque le processus va jusqu'au stade d'une marchandisation effective, est le fruit d'une transformation dans les conditions de production qui fait de l'activité universitaire du travail abstrait. C'est dans la monétisation du travail distincte de celle de la force de travail que réside le basculement dans le fonctionnement marchand.

À travers son abstraction, le travail universitaire est débarrassé de ses spécificités concrètes et toutes les activités - dans toutes les disciplines - sont rapportées à une dimension commune. 14 sur 20 
Dans l'évaluation REF des départements universitaires britanniques, tous les travaux évalués concourent à produire le score REF du département, et donc s'expriment dans la même unité. Tant que cette unité reste spécifique à l'exercice d'évaluation du travail académique, elle permet de quantifier la qualité sans pour autant en donner une valeur économique. C'est la dernière étape de l'évaluation REF, celle qui conduit à l'attribution des financements de la recherche, qui opère cette traduction en unité monétaire. Les scores REF des départements de toutes les universités britanniques d'une même discipline sont additionnés pour calculer l'étendu du «marché » national de la discipline, dans la terminologie du REF. Le rapport entre le score de chaque département et ce total est donc la «part de marché » emportée par ce département [Boulet et Harari-Kermadec, 2014]. La dernière étape d'abstraction efface totalement ce qui reste de différence disciplinaire en multipliant les «parts de marché » par un coefficient de coût disciplinaire (plus élevé pour la médecine et les disciplines expérimentales que pour les sciences humaines): une fois cette différence économique entre disciplines prise en compte, toutes les « parts de marché » sont directement commensurables. Elles donnent alors droit à une part de la subvention publique affectée à la recherche et obtiennent ainsi une expression monétaire. Le score REF de chaque département est ainsi converti en unité monétaire, en livres sterling en l'occurrence, parce qu'il se traduit mécaniquement en financement public. Cette transformation par des coefficients économiques produit une monétisation, une expression monétaire de la production scientifique du département. Traduite en monnaie, la production scientifique peut entrer dans la comptabilité de l'université à côté des volumes de frais d'inscription (qui représentent la production pédagogique) et des services annexes (consulting, location des locaux, ventes de produits dérivés). À leur tour, parce que la monnaie est l'équivalent général, toutes les productions de l'université sont mises en équivalence avec le reste de l'économie, ce qui suggère une substituabilité avec les autres biens et services marchands, mais aussi une logique commune de rentabilité. Le rapport coût/bénéfice qui préside à aux activités annexes à but lucratif des établissements peut alors s'étendre grâce à cette étape finale de commensuration monétaire aux activités académiques proprement dites, enseignement et recherche.

La monétisation joue aussi un rôle normalisateur [Jany-Catrice et Méda, 2013, p. 28, note 18] : si chaque caractéristique de l'enseignement supérieur a un équivalent monétaire, chaque département un chiffre d'affaires, il en découle des productivités qui appellent à être optimisées. L'exigence de retours sur investissements comparables aux benchmarks est alors naturalisée. Une complète adhésion n'est d'ailleurs pas nécessaire (comme pour le classement de Shanghai) pour que la transformation opère ; " les modalités contemporaines de la performance tendent à lui conférer un caractère prescripteur : elle apparaît comme un dispositif auquel personne ne croit tout à fait, mais auquel chacun (acteur individuel et collectif) répond et se plie » [Jany-Catrice, 2012, p. 28]. On observe ici un effet d'autorité de la quantification associée à une finalité directement économique. C'est ce qu'observe par exemple Boulet [2013, p. 60] à propos du 15 sur 20 
management d'une université anglaise dont les différents services sont directement comparés suivant leur « taux de profit ».

\section{Conclusion}

Les dispositifs d'évaluation et de classement dans l'enseignement supérieur structurent des transformations institutionnelle et organisationnelle, qui préparent une marchandisation. Marchandisation parce que ces dispositifs participent à instituer un marché de l'enseignement supérieur, mais aussi parce qu'ils sont repris par les établissements pour adapter leur fonctionnement organisationnel à cette nouvelle finalité. Ils donnent alors corps à une nouvelle forme sociale du travail universitaire, celle d'un travail abstrait, participant de la forme dominante de production sous le capitalisme, la production de marchandises. Le processus aujourd'hui reste incomplet, au moins en France, et perdure deux formes sociales concurrentes de la richesse produite à l'université. Deux modalités de validation sociale de la valeur coexistent, toutes deux monétaires, mais l'une marchande et l'autre non. Seule la première peut participer directement au processus de valorisation du capital.

Pour aller plus loin dans l'analyse de la cohabitation/concurrence entre ces deux formes de valorisation, il faudrait suivre au plus près les budgets des établissements. Le gouvernement français a d'ailleurs avancé d'un pas supplémentaire vers la marchandisation en programmant un remplacement des subventions publiques par des prises de participations dans les établissements " dans des conditions normales de marché » financier. Le troisième programme d'investissements d'avenir (PIA3) prévoie en effet d'appuyer (pour un total de 300 millions d'euros) les projets “ d'une dizaine de Territoires d'innovation de grande ambition ", le financement d'un projet prenant la forme « d'un apport au capital si les conditions financières, et notamment le modèle économique du projet, répondent aux critères d'investissement avisé » [Commissariat Général à I'Investissement et Caisse des Dépôts, 2017, p. 7]. II s'agit donc d'affecter des financements publics à la constitution d'un capital visant son accroissement, si l'on traduit le langage financier en termes marxiens. Un tel programme de recherche ferait nécessairement appel au droit et à la gestion, et relèverait du pluralisme scientifique revendiqué par la RFSE.

L'application du concept marxien de « forme sociale » pour caractériser ce processus d'insertion de l'enseignement supérieur dans le circuit du capital ouvre une nouvelle interrogation. Une évolution vers une autre forme sociale, à la fois abstraite et émancipatrice, est-elle possible ? $\mathrm{Si}$, dans le contexte du capitalisme contemporain, l'abstraction est une étape de la marchandisation, elle est tout aussi nécessaire dans un autre cadre pour permettre aux acteur.trices d'appréhender ensemble les différents éléments constitutifs de la richesse. Ce que Marx critique dans l'abstraction de la valeur d'échange, ce n'est pas tant en effet les abstractions 16 sur 20 
en soi qu'une mauvaise manière d'en faire : celle qui pose des équivalences forcées et surtout incontrôlées entre les objets et services qualitativement différents constituant la richesse. Critiquer la forme sociale que prend la richesse sous le capitalisme ne peut pas s'accompagner d'un projet de « retour » fantasmé à un état de la société où la richesse apparait sans médiation formelle [Postone, 1995]. En d'autres termes, la marchandisation de l'université est une modification de la forme, du mode d'existence de la richesse constituée par les services universitaires, mais il en existe d'autres, qui sont autant de formes non capitalistes de la richesse, qui décriraient les contours d'une organisation alternative de la production et de la distribution des biens et services. On peut les chercher dans les freins et les résistances au processus de marchandisation de l'université, dans les points aveugles des conventions marchandes, qui laissent ouvertes des alternatives.

\section{Références}

AMBROISE B. (2015). «Performativité de l'économie ou pouvoir symbolique ? » Revue Française de Socio-Économie, Hors-série n², p. 19- 35.

BARATS C., BOUCHARD J. et BIANQUIS HAAKENSTAD A. (éds.) (2018). Faire et dire l'évaluation: l'enseignement supérieur et la recherche conquis par la performance, Paris, France : Presses des Mines-Transvalor.

BARATS C. et LEBLANC J.-M. (2013). “ Généalogie de la co- construction médiatique du 'classement de Shanghai' en France. Corpus de presse et usages sociodiscursifs », Mots. Les langages du politique, $\mathrm{n}^{\circ} 102 / 2$, p. 67-83.

BELLOC B. (2003). Propositions pour une modification du décret 84-431 portant statut des enseignants-chercheurs. Rapport au ministre de la Jeunessse, de l'Education Nationale et de la Recherche.

BLANCHARD M. (2011) «Développer la recherche et maintenir la professionnalisation. Des Écoles Supérieures de Commerce françaises face aux transformations de l'enseignement supérieur européen ». Cahiers de la recherche sur l'éducation et les savoirs, n 3 p. 61-76.

BOULET E. (2013). “ La marchandisation de l'université à l'oeuvre. Le cas de l'université de Ghimwall, en Angleterre ». Mémoire de Master, Université Paris IV.

BOULET E. et H. HARARI-KERMADEC (2014). " Le rôle de la quantification dans le processus de marchandisation de l'université. étude de cas d'une université anglaise ", Politiques et management public, $\mathrm{n}^{\circ} 31 / 4$, p. 405-420.

BRUNO I. (2008). À vos marques $®$, prêts... cherchez! La stratégie européenne de Lisbonne, vers un marché de la recherche. Bellecombe-en-Bauges, France: Éd. du Croquant.

CHAUVEL S. et P. CLEMENT (2014). "Le rôle des palmarès et classements ", Savoir/Agir, $\mathrm{n}^{\circ} 29 / 3$, p. 35-42.

17 sur 20 
CALLON M., DESROSIERES A. et F. VATIN (éds.) (2013). Évaluer et valoriser: une sociologie économique de la mesure, Toulouse, France : Presses universitaires du Mirail.

Commissariat Général à I'Investissement et Caisse des Dépôts (2017). Programme investissements d'avenir. Appel à manifestation d'intérêt. Territoires d'innovation de grande ambition.

Cour des comptes (2018). Les droits d'inscription dans l'enseignement supérieur.

DELESPIERRE A. (2016) «L'internationalisation des grandes écoles d'ingénieurs françaises: une recomposition de la noblesse d'Etat ». Thèse de doctorat, Université Panthéon-Sorbonne Paris I.

DESROSIERES A. (1993). La politique des grands nombres: histoire de la raison statistique, Paris, France : Éditions la Découverte.

- (2008a). L'argument statistique ; 1 : Pour une sociologie historique de la quantification, Collection Sciences sociales (Paris). Paris, France : Mines ParisTech-les Presses.

- (2008b). L'argument statistique ; 2 : Gouverner par les nombres, Collection Sciences sociales (Paris),. Paris, France : Mines ParisTech-Les Presses.

DURAND C. (2014). Le capital fictif: comment la finance s'approprie notre avenir, Paris, France : les Prairies ordinaires.

ELOIRE F. (2010). "Le classement de Shanghai. Histoire, analyse et critique », L'Homme la Société, n¹78/4, p. 17-38.

ESPELAND W.N. et SAUDER M. (2007). «Rankings and Reactivity: How Public Measures Recreate Social Worlds », American Journal of Sociology, n¹13/1, p. 1-40.

- (2009). Rankings and diversity. Southern California Review of Law and Social Justice, 18, 587-608.

ESPERET, E. (2001). Nouvelle définition des tâches des enseignants et des enseignantschercheurs dans l'enseignement supérieur français. Rapport au Ministre de l'éducation Nationale.

FAURE, S., MILLET, M., \& SOULIE, C. (2005). Enquête exploratoire sur le travail des enseignants chercheurs. Vers un bouleversement de la "table des valeurs académiques»? Document de travail.

FLACHER D. et H. HARARI-KERMADEC (2013). "Tuition fees, self- esteem and social heterogeneity », Education Economics, n²1/2, p. 191-210.

FROUILLOU, L. (2014). Les écarts sociaux de recrutement des universités d'île-de-France: un processus de ségrégation? Espaces et sociétés, n 159(4), 111-126.

GINGRAS Y. (2013). Les dérives de l'évaluation de la recherche: du bon usage de la bibliométrie, Paris, France : Raisons d'agir.

HALL, R. et K. BOWLES. (2016). "Re-engineering higher education: the subsumption of academic labour and the exploitation of anxiety ». Workplace: A Journal for Academic Labor, $\mathrm{n}^{\circ}$ 28 , p. 30-47.

18 sur 20 
HARARI-KERMADEC H. et L. MOULIN. (2015). "Postface: De la mise en concurrence à la mise en marché de l'enseignement supérieur ». Formation emploi. Revue française de sciences sociales, $\mathrm{n}^{\circ} 132$ p. 91-103.

HARARI-KERMADEC $\mathrm{H}$. et M. LE BRETON (2013). « Engagement décennal ou endettement étudiant ? Les bi-admis ENS Cachan/HEC face au choix de leur école et de leur carrière ", Revue française de pédagogie, $\mathrm{n}^{\circ} 184$, p. 69-80.

HARRIBEY J.-M. (2013). La richesse, la valeur et l'inestimable : Fondements d'une critique socio-écologique de l'économie capitaliste. Français. Paris : Liens qui libèrent.

HARVIE, D. (2006). «Value Production and Struggle in the Classroom: Teachers within, against and beyond Capital », Capital \& Class, n³0/1, p. 1-32.

JANY-CATRICE F. (2012). «De l'évaluation des politiques publiques à la «performance totale» », Economie et institutions 18-19, p. 17-34.

JANY-CATRICE F. et D. MEDA (2013). " Les nouvelles mesures des performances économiques et du progrès social. Le risque de l'économicisme ", Revue du MAUSS, n41/1, $\mathrm{p}$. 371-397.

KARPIK, L. (2007). L'économie des singularités (Vol. 1-1). Paris, France: Gallimard.

LAMARCHE T. (2003). " L'OMC et l'éducation: normalisation de l'éducation pour en faire une marchandise échangeable internationalement? "Politiques et Management Public n²1/1, p. $109-130$.

LAVAL C., VERGNE, F., CLEMENT P. et G. DREUX (2012). La nouvelle école capitaliste, Paris, France : la Découverte.

LAZZARATO M. (2011). La fabrique de l'homme endetté : essai sur la condition néolibérale, Paris, France: Éd. Amsterdam.

LEBARON F. (2015). “ Injonction comptable et révolution culturelle à l'Université ». La nouvelle revue du travail [En ligne], $\mathrm{n}^{\circ} 6$.

MIN B. et R. FALVEY. (2018). «International Student Flows for University Education and the Bilateral Market Integration of Australia ». Higher Education n75/5, p. 871-89.

ORLEAN A. (2005). "La sociologie économique et la question de l'unité des sciences sociales ». L'Année sociologique, n55/2, p. 279-305.

PARADEISE, C. et J.-C. THOENIG (2015). In Search of Academic Quality. Palgrave Macmillan.

SAUDER M. et R. LANCASTER (2006). "Do Rankings Matter? The Effects of U.S. News \& World Report Rankings on the Admissions Process of Law Schools ", Law \& Society Review, $\mathrm{n}^{\circ} 40 / 1$, p. $105-134$.

SOBEL R. (2016). "Fétichisme de la marchandise et ontologie sociale chez Marx », Cahiers d'économie Politique / Papers in Political Economy, n70, p. 45-74.

SUPIOT A. (2015). La gouvernance par les nombres. Cours au Collège de France, 2012-2014, Nantes, France : Institut d'Études Avancées de Nantes.

19 sur 20 
SZADKOWSKI, K. (2016). «Towards an Orthodox Marxian Reading of Subsumption(s) of Academic Labour under Capital ». Workplace: A Journal for Academic Labor, n 28, p. 9-29.

THEVENOT L. (1989). «Equilibre et rationalité dans un univers complexe ». Revue économique, 147-197.

VERDALLE L. de, VIGOUR C. et T. Le Bianic (2012), "S'inscrire dans une démarche comparative », Terrains travaux, $\mathrm{n}^{\circ} 21 / 2$, p. 5-21.

VINOKUR A., \& EYRAUD, C. (2017). Le "Higher Education and Research Act 2017 »: acte de décès du service public de l'enseignement supérieur en Angleterre ? Document de travail. 